\title{
UNA APROXIMACIÓN FENOMENOLÓGICA A LA EXPERIENCIA DE RECAÍDA EN EL CONSUMO DE ALCOHOL EN PERSONAS CON SÍNTOMAS DISOCIATIVOS Y EXPERIENCIAS TRAUMÁTICAS
}

\author{
A PHENOMENOLOGICAL APPROACH TO THE EXPERIENCE OF RELAPSE \\ IN ALCOHOL CONSUMPTION IN PEOPLE WITH DISSOCIATIVE SYMPTOMS \\ AND TRAUMATIC EXPERIENCES
}

\author{
Nicolás Matteo-Guzmán* \\ Universidad Adolfo Ibáñez \\ Santiago-Chile \\ David Martínez-Pernía \\ Universidad Adolfo Ibáñez \\ Santiago-Chile
}

Recibido marzo 2020/Received March 2020

Aceptado junio 2020/Accepted June 2020

\begin{abstract}
RESUMEN
El objetivo de esta investigación fue indagar en la recaída del consumo de alcohol en personas que sufrieron experiencias traumáticas y síntomas disociativos. Se realizaron seis entrevistas, basadas en la fenomenología trascendental de Edmund Husserl, en las que se indagó en la experiencia de recaída por consumo de alcohol. El método de análisis de entrevistas se realizó según la propuesta de fenomenología descriptiva de Giorgi. Los resultados de este estudio dan cuenta de una estructura temporal en la experiencia de recaída. En la fase inicial la persona tiene recuerdos asociados a la experiencia traumática, lo que le lleva a comenzar el consumo de alcohol para aliviar el sufrimiento experimentado. Posteriormente, y mientras se produce el consumo, siente momentos fugaces de satisfacción, los que son reemplazados por la sensación de pérdida de control y una valoración negativa de sí mismo. El fin de la recaída llega cuando la persona recurre a su entorno para dar cuenta de lo sucedido. Este estudio muestra la relevancia que tiene la temporalidad en la experiencia de recaída en el consumo de alcohol. Además, se propone que el fenómeno de la disociación química atribuido al alcohol y el acceso a la carga emocional es un proceso alternante y dependiente de la temporalidad.
\end{abstract}

Palabras Clave: Recaída, Alcohol, Disociación, Fenomenología, Experiencia traumática.

\section{SUMMARY}

This research aims to investigate the relapse in alcohol consumption in people who suffered traumatic experiences and dissociative symptoms. Six interviews were conducted based on the transcendental phenomenology of Edmund Husserl, in which the experience of relapse due to alcohol consumption was investigated. The interview analysis method was conducted according to Giorgi's descriptive phenomenology proposal. The results of this study show a temporary structure in the relapse experience. In the initial phase the person has memories associated with the traumatic experience which leads him or her to start drinking alcohol to relieve the suffering experienced. Subsequently, and while consumption occurs, he or she feels fleeting moments of satisfaction, which are replaced by the feeling of loss of control and a negative assessment of himself or herself. The end of the relapse comes when the person resorts to his or her environment to explain what happened. This study shows the relevance of temporality in the experience of relapse in alcohol consumption. Furthermore, it is proposed that the phenomenon of chemical dissociation attributed to alcohol and access to emotional burden is an alternating and temporality-dependent process.

Key Words: Relapse, Alcohol, Dissociation, Phenomenology, Traumatic Experience. 


\section{INTRODUCCIÓN}

Un elemento teórico relevante en el estudio de adicción a las drogas es la distinción entre tolerancia, dependencia y abstinencia. El primero de ellos hace referencia a la habituación generada hacia la sustancia consumida, lo que se traduce en la disminución de la efectividad del efecto según la repetición de la exposición a la misma (Domjam, 2010; OMS, 2008). El segundo da cuenta del estado de necesidad de repetir el consumo, teniendo o no noción de las consecuencias, pudiendo entenderse como un intento de reducción de una reacción aversiva posterior al consumo (Domjam, 2010; OMS, 2008). El tercero, y último, es la abstinencia. Este concepto hace referencia a la privación del consumo de cualquier sustancia, independientemente de la motivación por la que se produce (OMS, 2008). Una de las formas en las que puede derivar la abstinencia es en lo que se conoce como Síndrome de Abstinencia. Este es un conjunto de síntomas variables tanto en su intensidad como en su grado de compromiso fisiológico, el que se presenta cuando se suspende o reduce la exposición a la sustancia psicoactiva que se consumió de forma repetida (OMS, 2008).

Diversos estudios han relacionado el mantenimiento de un patrón de consumo problemático-dependiente de drogas con la presencia de eventos traumáticos en la vida de las personas, específicamente aquellos sucedidos en la infancia (Bastos et al., 2017; Gielen, Havermans, Tekelenburg y Jensen, 2012). Un estudio realizado por Konstenius et al. (2017) con 1.274 pacientes adultos en tratamiento ambulatorio o con hospitalización por uso de sustancia, mostró una alta prevalencia en la comorbilidad de ambas categorías. Del total de los evaluados 53,6\% informó al menos una experiencia traumática en la infancia, siendo más significativas aquellas ligadas al abuso emocional $(38,3 \%)$, al abuso físico $(28,6 \%)$ y la violencia en la familia $(27,7 \%)$. En esta misma línea, en un estudio realizado por Langeland, Draijer y Van den Brink (2002) con sujetos que buscaban tratamiento por consumo de alcohol se observó que cerca del $24 \%$ reportó abusos sexuales en su infancia, así como también ser testigos de violencia doméstica (16\%) y abuso físico (15\%). Asimismo, un estudio que contempló a 423 sujetos que recibían tratamientos por trastorno por uso de sustancias arrojó que el 97,4\% de estos experimentaron algún tipo de experiencia potencialmente traumática a lo largo de su vida, mientras que de este total el 36,6\% cumplió con criterios para ser diagnosticados con un Trastorno de Estrés Postraumático (en adelante TEPT) (Gielen et al., 2012). Debido a estos antecedentes es relevante señalar la importancia que tienen las vivencias traumáticas en sujetos que presentan consumo problemático de sustancias. De estas experiencias traumáticas especial atención deben ponerse a las experiencias traumáticas de la infancia, las que deben ser integradas en el proceso terapéutico (Van Den Brink, 2015).

Gielen et al. (2016), siguiendo con la relación entre estas variables, presentaron un estudio cualitativo en el que se dio cuenta de que la presencia de eventos traumáticos tenía mayor impacto en el mantenimiento de una conducta de consumo problemático. Los mismos autores observaron que dentro de los motivos atribuidos al consumo de la sustancia destacan: motivaciones sociales, por los efectos propios de la sustancia, por expansión mental o de conciencia, por motivos de mejora anímica y como forma directa de evasión o enfrentamiento de dificultades (Gielen et al., 2016).

De forma complementaria se comenzó a dar cuenta del papel que juegan los síntomas disociativos en la relación establecida entre las experiencias traumáticas y el consumo de alcohol. En esta línea, Lyssenko et al. (2017) señalaron que la presencia de síntomas disociativos da cuenta de un curso diferente y más complejo en la comprensión de la relación entre el consumo de sustancias y el trauma. A su vez, también juega un rol importante al conceptualizar y definir el TEPT y su subtipo específico (APA, 2013).

En los resultados planteados por las investigaciones que integran estos tres elementos (consumo, disociación y trauma) se observaron algunas diferencias en lo que respecta a la presencia o ausencia de la disociación. Estudios en los que dicha dimensión se presentó baja, junto con altos niveles en lo que respecta al uso de sustancias y la presencia de eventos traumáticos, se planteó la hipótesis de la "Disociación Química", la que vendría a ser una alternativa frente a la capacidad limitada de disociarse en términos psicológicos, la que podría generar un estado similar a causa del potencial evasivo, calmante o adormecedor atribuido a la sustancia. Por tanto, la conducta de abuso se planteó como un método que ayuda a manejar las experiencias traumáticas experimentadas a lo largo de la vida (Najavits y Walsh, 2012; Klanecky, 
Harrington y McChargue, 2008; Schäfer et al., 2007; Langeland, Draijer y Van Den Brink, 2002). Dicha relación ha sido demostrada en sujetos con consumo problemático o dependencia al alcohol, marihuana y benzodiacepinas (Klanecky, Harrington y McChargue, 2008; Langeland, Draijer y Van Den Brink, 2002; Schäfer et al., 2007). Además, cuando se observaron altos niveles de estas tres dimensiones (conducta de abuso, disociación y trauma) hubo una tendencia a presentar la sustancia como un medio que permite el acceso a emociones o recuerdos vinculados a las experiencias traumáticas (Evren et al., 2011; Mergler et al., 2017; Schäfer et al., 2007; Van Den Bosch, Verheul, Langeland y Van Den Brik, 2003), estableciéndose mayor conexión con aquellas ligadas al abuso emocional y la negligencia física (Evren et al., 2011; Najavits y Walsh, 2012). Los antecedentes recientemente revisados muestran que la presencia de síntomas disociativos en personas con TEPT producen un curso más severo de problemas asociados al consumo de sustancias, específicamente por el uso de opiáceos o analgésicos, seguido por una mayor cantidad de episodios de sobredosis a lo largo de la vida (Mergler et al., 2017), sugiriendo entonces un rol mediador entre la presencia de trauma/TEPT y el uso de sustancias (Evren et al., 2011).

Gran parte de estas investigaciones fueron de tipo transversal, retrospectivas y centradas en las experiencias traumáticas tempranas (Evren $e t$ al., 2011; Klanecky, Harrington y McChargue, 2008; Langeland, Draijer y Van Den Brink, 2002; Mergler et al., 2017; Najavits y Walsh, 2012; Schäfer et al., 2007; Van Den Bosch, Verheul, Langeland y Van Den Brik, 2003). Respecto de la comprensión de los síntomas clínicos, se observó una distinción entre la sola presencia de síntomas disociativos (Evren et al., 2011; Najavits y Walsh, 2012; Schäfer et al., 2007) y una forma comprensiva de TEPT de tipo disociativo, integrada en la quinta versión del Manual diagnóstico y estadístico de los trastornos mentales (DSM-V, por sus siglas en inglés) que viene proporcionada por la presencia de síntomas de despersonalización y desrealización (APA, 2013; Mergler et al., 2017). Mientras tanto, en lo que guarda relación con la descripción del uso de sustancias se observaron diferencias entre el consumo de alcohol (Evren et al., 2011; Klanecky, Harrington y McChargue, 2008; Langeland, Draijer y Van Den Brink, 2002; Schäfer et al., 2007) y el de drogas en general
(Mergler et al., 2017; Najavits y Walsh, 2012; Tamar-Gurol et al., 2008; Van Den Bosch, Verheul, Langeland y Van Den Brik, 2003).

Si bien la evidencia científica natural da cuenta de la importancia y la correlación estadística entre estos tres elementos (conducta de abuso, disociación y trauma), la evidencia científica que existe respecto de la experiencia subjetiva (fenomenológica) de recaída en el consumo de alcohol ha pasado altamente inadvertida. Dicho esto, la presente investigación buscará indagar en la experiencia de recaída por consumo de alcohol en sujetos que den cuenta de experiencias traumáticas y con síntomas disociativos. La relevancia de estudiar este fenómeno desde la experiencia de la persona que recae en el consumo es la de lograr una mejor y más detallada comprensión del fenómeno de la recaída y a su vez nutrir los procesos terapéuticos que emerjan de esta comprensión.

\section{METODOLOGÍA}

\section{Tipo y abordaje del estudio}

Se realizó un estudio fenomenológico basado en la perspectiva transcendental de Edmund Husserl para indagar en la experiencia de los participantes en los momentos antes, durante y después de la recaída del consumo de sustancias. Esta perspectiva recoge la descripción de la experiencia del sujeto tal y como la vive (Aguirre-García y Jaramillo-Echevarri, 2012; Giorgi, Giorgi y Morley, 2017), entregando "un conocimiento de la naturaleza esencial de lo Real" (Husserl, 1931, p.45). En palabras de Clark Moustkas, el objetivo de esta tradición filosófica es "describir las cosas en sí mismas (...) y que sean entendidos sus significados y esencias a la luz de la intuición y la autorreflexión" (1994, p. 27).

\section{Participantes}

Se definieron como criterios de inclusión a hombres y mujeres mayores de 18 años, diagnosticados con trastorno por uso de sustancias, que presenten síntomas disociativos y eventos vivenciados como traumáticos en algún momento de su vida, y que hayan sido derivados para recibir tratamiento por consumo de alcohol en el Centro de Salud Mental Comunitaria de la ciudad de Talca. Los criterios de exclusión fueron la presencia de sintomatología psicótica, ideación suicida activa y que tuvieran antecedentes judiciales activos al momento de la recolección de los datos. 


\section{Muestreo y muestra}

El muestreo fue realizado mediante muestras homogéneas (Hernández, Fernández y Baptista, 2010). Considerando que el objetivo central de la investigación es comprender y profundizar en la fenomenología de los participantes, es decir, desde una perspectiva desde dentro (Krause, 1995; Hernández, Fernández y Baptista, 2010), es que el tamaño muestral estuvo definido en función del punto de saturación teórica (Martínez-Salgado, 2012).

\section{Recolección de datos}

La recolección de los datos se realizó mediante entrevistas en profundidad desde una aproximación fenomenológica. La entrevista fenomenológica tiene como característica esencial la recolección de datos del "qué" de la experiencia y el "cómo" es vivida por la persona (Moustakas, 1994). Este tipo de entrevista fenomenológica comienza con un ejercicio de atención estabilizadora en donde se busca contextualizar y mantener el enfoque atencional en el proceso de entrevista (Petitmengin, 2006; Silva-Mack, Araya-Véliz, Martínez-Pernía, y Arístegui, 2018). Debido a que el objetivo de la entrevista era indagar la experiencia en los momentos antes, durante y después de la recaída del consumo de sustancias se comenzó la entrevista con preguntas de carácter situacional: ¿cuándo sucedió?, ¿dónde estabas?, ¿con quién estabas?, ¿qué estaba sucediendo en ese momento?, ¿cómo notas lo que está sucediendo?, ¿cómo notas que esto es una recaída? Una vez contextualizada la situación se indagó detalladamente en los aspectos experienciales y su temporalidad: ¿cómo fueron sucediendo los hechos?, ¿qué sucedió primero?, ¿cómo comienzan a ocurrir las cosas?, ¿cómo te sientes con lo que está pasando? Estos aspectos de la experiencia son denominados las dimensiones sincrónicas (en un momento particular) y diacrónicas (la temporalidad) de la experiencia (Olivares, Vargas, Fuentes, Martínez-Pernía, Canales-Johnson, 2015; Petitmengin, 2006).

Las entrevistas fenomenológicas fueron audiograbadas, transcritas, analizadas y almacenadas por el investigador principal. Las entrevistas fueron realizadas entre febrero y noviembre de 2019 teniendo una duración aproximada de 30 minutos cada una.

\section{Análisis de datos fenomenológicos}

$\mathrm{El}$ análisis de los datos fenomenológicos se realizó mediante el método psicológico-fenomenológico propuesto por Amedeo Giorgi (Giorgi, Giorgi y Morley, 2017). Esta metodología elude la interpretación tanto en el periodo de recolección de los datos como en su posterior análisis. Para ello el investigador debe ocuparse de analizar las experiencias vividas del participante asumiendo una postura de reducción fenomenológica y la experiencia de "epoché" (Merriam, 2009; Moustakas, 1994), evitando generar una posición personal acerca de la posible existencia de objetos que se dan en la conciencia (Aguirre-García y Jaramillo-Echevarri, 2012; Giorgi, Giorgi y Morley, 2017).

El análisis de datos según el método de Giorgi está descrito en cinco grandes pasos (Giorgi, Giorgi y Morley, 2017):

1. Lectura de todas las descripciones proporcionadas por los participantes.

2. Asumir una actitud de reducción fenomenológica-psicológica, la que hace referencia a que los objetos que emergen dentro de la descripción se consideran fenómenos que se presentan a la conciencia del experimentador. Esta actitud se mantiene durante todo el proceso, con la finalidad de realizar un análisis basado en la descripción de la experiencia del entrevistado y no en los juicios o percepciones del entrevistador (Hamilton, Pernía, Puyol, Dell' Aquila, 2019).

3. La descripción es dividida en diferentes Unidades de Significado, debiendo identificarse los lugares en el relato en donde estas cambian o establecen una diferencia relativamente significativa.

4. Se deben transformar las Unidades de Significado proporcionadas por el sujeto en expresiones que resalten los significados de su experiencia.

5. Se realiza una descripción de la estructura fenomenológica de la experiencia basada en las unidades de significado transformadas, la que agrupa a la totalidad de la muestra del estudio.

\section{Criterios éticos}

La presente investigación cuenta con la aprobación tanto del Comité de Bioética del Servicio de Salud del Maule como del Comité de 
Ética de la Universidad Adolfo Ibáñez. A cada participante se le entregó un consentimiento informado, el que fue firmado y adjuntado al expediente clínico perteneciente a su centro de tratamiento. Paralelamente se resguardó la privacidad de cada uno de los usuarios, para esto se asignó un número a cada una de las entrevistas, más la fecha en la que fue realizada.

\section{RESULTADOS}

El punto de saturación teórica de este estudio se produjo en la realización de seis entrevistas ( 3 hombres y 3 mujeres, con edades entre 54 y 28 años), a partir de estas se obtuvo un particular nivel de detalle de la experiencia de recaída y donde la información dejó de generar nuevas propiedades y categorías experienciales.

El análisis de las entrevistas propició la aparición de 4 categorías temporales de análisis que serán descritas a continuación:

1. Antecedentes: da cuenta de la carga emocional y cognitiva previa a la experiencia, prima un malestar emocional constante centrado en la pena, la rabia, más la sensación de desesperación y desesperanza frente a la situación actual. Elementos que se entrecruzan con la evocación de la experiencia traumática a causa de la realidad del contexto. En esta fase temporal es el momento donde se da curso a la planificación de la recaída, siendo esta una acción que permitirá controlar el malestar general sostenido por un continuo de tiempo. S1: Siento que todo lo que pasó es culpa mía, y sé que no es así, pero igual.

S2: Fui a buscar ayuda donde corresponde, se supone, a nuestros jefes. Y doble es el maltrato, me humillaron mucho, me trataron muy mal, muy mal. El hecho de que me pasen cosas graves y yo no tenga a nadie a quien acudir ha marcado toda mi vida. Sí, porque me siento sin una identificación como tienen todos los seres humanos.

S3: Empieza el cuestionamiento de si estoy haciendo bien las cosas. Si vale la pena lo que estoy haciendo. Qué voy a hacer de aquí en adelante.

S5: Es todo lo que me estaba pasando... sentirme estancada, como sin alas. Sentirme también como poca cosa, como que no estoy brillando por mí misma.
S6: Mmm más que una pena tenía mucha rabia. Como una impotencia. Fue una situación que pah... eso.

2. Inicio del consumo: Es el momento en que colapsa la capacidad de tolerar el malestar y el escenario actual se vuelve insostenible. La persona toma la decisión de dar inicio al consumo planificado, el que se desarrolla en una circunstancia particular y en un lugar predeterminado. Conductualmente se describe un consumo voraz que debe apagar la angustia y dar paso a un alivio rápido de ciertos malestares que a ratos se han tornado inespecíficos.

S1: Siento que la medicación ya no está haciendo efecto. Ya no estoy pudiendo conciliar el sueño... No quiero salir a ningún lado, no quiero ver el exterior, mi mundo es mi pieza... Me da ansiedad y me las tomo muy rápido. Te juro que lo único que quiero es quedarme dormido. No pensar más. No pensar en ningún problema.

S2: Los sentimientos en mí provocan que beba más o que beba menos dependiendo de la profundidad del factor externo al que yo me pueda someter... La desesperación de no querer estar, y no querer seguir sintiendo más pena. Ya basta... Tengo una ansiedad. Una ansiedad que debe ser bebida.

S3: Yo ya tenía todo programado dentro de mi mente. Sí, yo tenía todo lo que iba a hacer.

3. Efectos de la recaída: la carga emocional inicial comienza a cambiar debido a la influencia del alcohol. Se experimentan diversas consecuencias conforme el tiempo avanza, las que tienden a ser recursivas acerca de la comprensión de la recaída. En un primer momento se da cuenta de cómo se transita desde el malestar a una experiencia más placentera, facilitadora de bienestar, la que mutará, conforme pase el tiempo, a nuevos cuestionamientos respecto de la acción que llevarán a revalorar el tratamiento y las diferentes implicancias de lo sucedido.

S1: Es un consumo corto, que lo hago, como tú me dices, narcotizante, para poder dormir y pasar la pena... Cuando estoy ya medio caramboliao, medio mareado, siento que está mi niñita chica, ahí al lado. 
S3: (Beber) me gratifica. Me hace sentir; de hecho, me olvido de los problemas, sabiendo que después van a venir muchos más a consecuencia de. Pero me hace como evadir por un tiempo.

S4: Tenía que elegir. Ya había tenido meses de abstinencia antes de esa última recaída, y lo había pasado bien, y el día después de la recaída, como muchas veces hice el análisis: voy a perder la pega, mil cosas, la salud. Y a lo mejor, ¿iré a encontrar otra mujer? O bien, me iba a quedar alcoholizado.

S5: Es que el alcohol me relaja y hace que viva instantes de felicidad... La ansiedad como que se olvida. Se va la ansiedad y queda solo pasarlo bien... Se supone que iba a ser controlado. Eran unos cortitos hasta cierto punto, pero después me descontrolé.

S6: Uno sabe lo que va a pasar, pero en ese minuto uno no lo asume y es como hacerse una trampa a uno mismo. Como hacerse trampa jugando solitario.

4. Socialización de lo sucedido. El fin de la recaída: con la constancia de los cuestionamientos personales respecto de lo que ha sucedido, el sujeto comienza a compartir con su entorno más cercano la recaída, limitando el acceso al alcohol para después concretarse el término de la recaída.

S2: Desgraciadamente tengo que reconocer que si no hubiera sido por él (hijo) yo no estaría... Yo pensé que siempre me iba a quedar así, con crisis, no podía, aparte de que no entendía nada.

S3: Le he fallado varias veces a mi hijo, a toda mi familia en realidad. Entonces cuando se tiene la lucidez se da cuenta de que la está embarrando. No para con la cuestión. No para. Va a terminar mal.

S5: Me da lata que mi mamá y mi hermano pasen por esas cosas. Siento que yo soy la mayor, que debía dar el ejemplo y hago puras cagás.

\section{Estructura de la experiencia fenomenológica}

Mediante la generación de estas unidades de significado se obtuvo la siguiente estructura fenomenológica de la experiencia de consumo (Figura 1).

Previo al consumo de alcohol sienten una creciente carga emocional negativa, prima la pena, la angustia, la rabia y en ocasiones la vergüenza debido a los recuerdos de diferentes experiencias que ellos mismos han calificado como traumáticas. Memorias que han emergido mediante la exposición a diferentes situaciones ligadas de alguna manera a los eventos vivenciados. Con esto cuestionan su capacidad, así como la de su entorno, de poder tolerar este malestar. La persona se conecta con una incertidumbre de su propio futuro, dudando de la utilidad que tiene estar intentando controlar el consumo y mantener la abstinencia.

Poco a poco las personas comienzan a aceptar y rendirse al consumo. La necesidad de lidiar con el intolerable malestar hace que la posibilidad de

\section{Figura 1}

La presente figura da cuenta del proceso de la recaída resaltando el momento de aparición de cada una de las categorías temporales de la experiencia

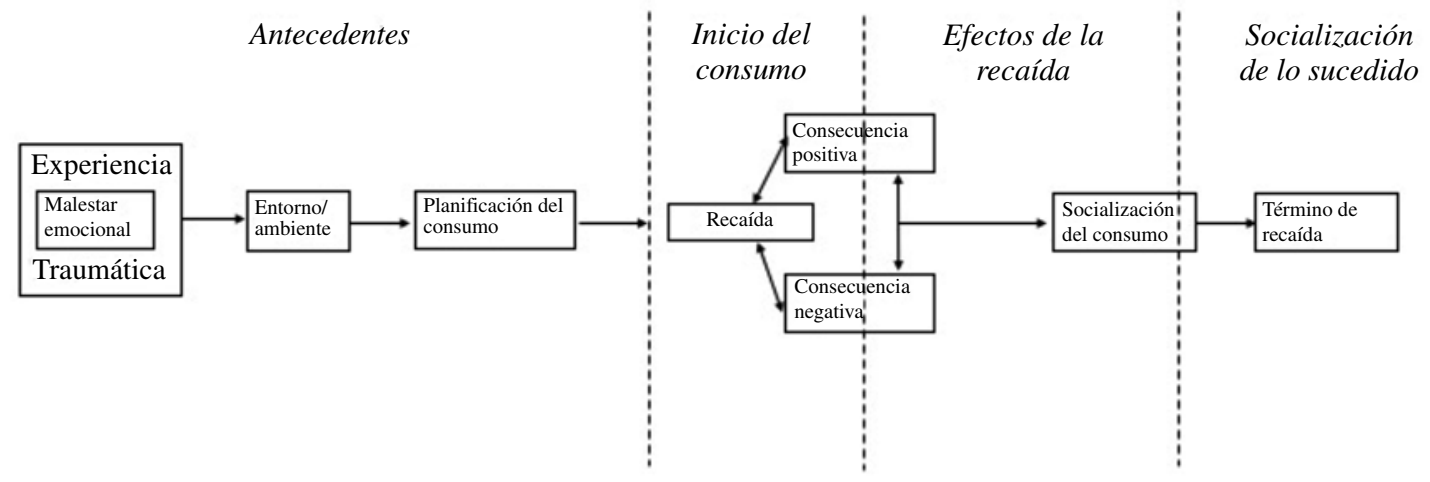


mejoría solo se encuentre en esta acción, por lo que la planificación de consumo se construye con la intención de no dañar de manera absoluta lo conseguido en el tratamiento. Sin embargo, conforme con la recaída se desarrolla la voracidad y compulsión dominan la experiencia, tanto el malestar como el cuerpo se comienzan a controlar y poco a poco se va desdibujando lo planificado. El mantenerse en la recaída comienza a ser una posibilidad real, llegando incluso a contemplar la opción de utilizar otro tipo de sustancias. Hasta este momento el efecto es positivo y controlado.

Según el tiempo avanza el escenario cambia, se transita a una nueva idea de pérdida de control, que vuelve a conectar las emociones con la pena, el miedo y la angustia, las que esta vez han sido activadas por otro tipo de eventos pero que vuelven a impactar en la valoración negativa de sí mismo y de sus acciones. A su vez, el consumo va en un aumento que se relaciona con la fugacidad de los momentos de bienestar, los que se van diluyendo con los juicios emitidos hacia sí mismos. En este punto el entorno es visto como un recurso que puede ayudar en el control de la recaída, por lo que se socializa lo que está sucediendo mientras invaden sentimientos de vergüenza y culpa.

\section{DISCUSIÓN}

El objetivo central propuesto para el presente estudio era la comprensión de la experiencia subjetiva de recaída de aquellos sujetos que presentan consumo de alcohol, antecedentes de experiencias traumáticas y síntomas disociativos. Los resultados dan cuenta de una estructura de la experiencia constituida por 4 categorías temporales y que a su vez integran significados particulares: (1) Antecedentes: momento en que emergen los recuerdos de la experiencia traumática, se activa la carga emocional y se planifica el consumo. (2) Inicio del consumo: constituye el momento específico de la recaída y la integración de los efectos conseguidos. (3) Efectos del consumo: contiene el tránsito por los efectos de la recaída, describiéndose eventos mediatos e inmediatos. (4) Socialización de lo sucedido: prima el intento de controlar el consumo y poner fin a la recaída con la ayuda del entorno.

Un elemento central en la comprensión del fenómeno de la recaída para efectos de este estudio se relacionó con la necesidad de modificar un estado emocional negativo ligado a la memoria traumática, lo que vendría a propiciar el inicio de la recaída. Este resultado condice con lo señalado en otras investigaciones que sostienen que la aparición de recuerdos de eventos calificados como potencialmente traumáticos está relacionado con la mantención de una conducta adictiva, así como también con una mayor predisposición a la recaída o a los eventos de sobredosis (Bastos et al., 2017; Mergler et al., 2017; Gielen et al., 2016).

Respecto de los motivos que inducen al inicio del consumo, los resultados coinciden con lo propuesto por Mau, Muller y Kaya-Roessler (2018), los que dan cuenta que el uso del alcohol durante la recaída tiene que ver con una forma de autorregularse unido con la experiencia de estar desconectado de los otros. Asimismo, Gielen et al. (2016) sostienen que el uso de sustancias en sujetos que han presentado experiencias traumáticas se relaciona con una continua búsqueda de potenciar un estado de euforia. Además de una forma de afrontamiento que ayude tanto a evadir como a enfrentar las dificultades ligadas a la memoria traumática (cognitivas y emocionales). Es importante destacar que en este punto emerge un elemento que es relevante de considerar y que guarda relación con la temporalidad del efecto.

Estudios previos que dan cuenta de las posibles implicancias del consumo y la recaída han persistido en la explicación centrada en la correlación entre variables, dejando de lado la dimensión temporal del proceso de la recaída (Mergler et al., 2017; Gielen et al., 2016; Evren et $a l ., 2011)$. En esta línea se observa un desarrollo en la comprensión de este fenómeno en dos formas independientes: 1) la hipótesis de la disociación química: concepción en donde la sustancia ayuda a catalizar un potencial evasivo y calmante, propiciando un efecto más inmediato, y que está presente en sujetos con consumo de alcohol (Najavits y Walsh, 2012; Klanecky, Harrington y McChargue, 2008; Schäfer et al., 2007; Langeland, Draijer y Van Den Brink, 2002). 2) La sustancia de abuso es un medio que posibilita el acceso al contenido emocional asociado a la experiencia traumática (Evren et al., 2011; Mergler et al., 2017; Schäfer et al., 2007; Van Den Bosch, Verheul, Langeland y Van Den Brik, 2003). Sin embargo, y al contrario de esta literatura que presenta ambos fenómenos como procesos independientes, en este estudio se presentan resultados en que el sujeto tiende a alternar entre estas dos posiciones y que es la temporalidad la que sostiene este tránsito. Una 
posible interpretación de esta alternancia es que al comenzar el consumo emerge rápidamente una sensación de bienestar, ligada a la evasión o a la disociación de la experiencia emocional negativa. Sin embargo, conforme avanza el tiempo, es posible que el sujeto integre la noción del contexto y se retome el contacto con el contenido emocional inicial, debiendo aumentar el consumo para volver a conectar con la sensación inicial. Al parecer, son la necesidad de recuperación y la socialización del consumo los elementos que potencian el fin de la recaída e interrupción de la circulación entre los tipos de efectos (inmediatos y mediatos).

Desde la filosofía y la revisión de la teoría fenomenológica se encontró apoyo teórico que sostiene esta interpretación de alternancia en las consecuencias y los efectos que la recaída tiene para la persona. Es así como Ricoeur (en Odland, Asplund y Nergaard, 2015) sugiere que por medio de la conciencia es que el pasado se hace presente en forma de recuerdo, siendo el presente una percepción directa que se conecta con la conciencia, mientras que lo referente al futuro es la vinculación con las expectativas de un elemento en particular. Sin embargo, la vuelta al pasado no permite modificar aquello que ha sucedido en términos de experiencia, pero es posible, mediante la realización de una epoché, modificar el sentido y la carga asumida por lo ocurrido, proceso descrito por medio del concepto de rememoración (Castillo, 2010). Lo anterior se sostiene en la premisa de que esto está ligado con la posibilidad de narrar y articular diferentes momentos temporales (Lythgoe, 2018) asumiéndose una tensión entre lo temporal y lo narrativo. Por tanto, el tiempo en sí se articula en lo narrativo, y a su vez la narración es significativa cuando se describen elementos de la experiencia temporal (Lythgoe, 2018). Es entonces de particular importancia llevar estos elementos emergidos de un modo prerreflexivo en la experiencia de consumo al plano del lenguaje, a lo reflexivo, y así dotarlos de sentidos mediante la narración.

En sí misma la entrevista articulada en el presente estudio da cuenta de que estas memorias puedan tomar parte en la narrativa, el traspaso del "qué" al "cómo", en el sentido procesal de la experiencia (Petitmengin, Bitbol y Ollagnier, 2015), lleva a que se adentre en la fenomenología de la memoria, en forma de mneme y "anamnesis", respectivamente (Perkowska, 2013). El primero de estos elementos refiere a la evocación simple, muchas veces pasiva, mientras que la anamnesis, referida al "cómo" busca en las huellas del recuerdo, la lucha contra el olvido es en sí la forma de rememorar aquellos elementos que han sido borrados (Perkowska, 2013). Se observa entonces que esta forma de indagar en la experiencia supone un insumo nutritivo en contenidos que permiten articular un proceso de acompañamiento psicoterapéutico que permita abarcar el consumo de alcohol y su relación con los eventos traumáticos y los síntomas disociativos.

\section{CONCLUSIÓN}

El presente estudio resulta ser un aporte para la comprensión de la recaída en sujetos con síntomas disociativos y experiencias traumáticas. Entrega una comprensión fenomenológica a un fenómeno que ha tenido un desarrollo comprensivo al alero de las ciencias naturales. Aporta además al estudio de una problemática transversal en la población consultante de tratamientos por salud mental.

La investigación desarrollada presenta ciertas limitaciones en lo que responde a aspectos metodológicos. En primer lugar, se asume la utilización de una sola sustancia de abuso, omitiendo la posibilidad de policonsumo, así como también la posibilidad de comorbilidad con otros cuadros psiquiátricos. Por otra parte, es un estudio exploratorio con alcances descriptivos que propone la posibilidad de seguir profundizando en el estudio de estas variables, así como en la fenomenología de la recaída. Se sugiere para futuras investigaciones el profundizar en la comprensión de la temporalidad en la recaída y específicamente en las consecuencias que manifiestan los consumidores, más aún cuando se encuentran en un proceso de tratamiento, resultando importante considerar tanto el tiempo de este cómo la motivación en el mantenimiento del mismo. 


\section{REFERENCIAS}

Aguirre-García, J. y Jaramillo-Echeverri, L. (2012). Aportes del método fenomenológico a la investigación educativa. Revista Latinoamericana de Estudios Educativos, 8(2), 51-74.

American Psychiatric Association (2013). DSM-V: Diagnostic and statistical manual of mental disorders. American Psychiatric Publishing.

Bastos, C., Lasiuk, G., Barton, S., De Fatima, M. y Da Silva, E. (2017). An exploration of addiction in adults experiencing early-life stress: a metasynthesis. Revista Latinoamericana de Enfermagem, 25. doi: 10.1590/1518-8345.2026.2939.

Castillo, M. (2003). Sobre la rememoración y la política: Tensiones entre W. Benjamin y P. Ricoeur. II seminario internacional políticas de la memoria. Centro Cultural de la Memoria Haroldo Conti.

Domjam, M. (2010). Principios de aprendizaje y conducta. Santa Fe: Wadsworth Cengage Learning.

Evren, C., Sar, V., Dalbudak, E., Cetin, R., Durkaya, M., Evren, B. y Celik, S. (2011). Lifetime PTSD and quality of life among alcohol-dependent men: impact of childhood emotional abuse and dissociation. Psychiatry research, 186, 85-90. doi: 10.1016/j. psychres.2010.07.004

Gielen, N., Havermans, R., Tekelenburg, M. y Jensen, A. (2012). Prevalence of post-traumatic stress disorder among patients with substance use disorder: it is higher than clinicians think it is. European Journal of Psychotraumatology, 3 (1), 1-9.

Giorgi, A., Giorgi, B., Morley, J. (2017). The Descriptive phenomenological psychological method. In C. Willing y W. Stainton Rogers (Eds.) The Sage handbook of qualitative research in psychology ( $2^{\text {nd }}$ ed., pp. 176-192). Sage.

Hamilton, A., Pernía, D., Puyol-Wilson, C. y Carrasco Dell'Aquila, D. (2019). What makes metalheads happy? A phenomenological analysis of flow experiences in metal musicians. Qualitative Research in Psychology, 16 (4), 537-565.

Hernández, R., Fernández, C. y Baptista, P. (2010). Metodología de la investigación. McGraw Hill.

Husserl, E. (1931). Ideas. George Allen and Unwin.

Klanecky, A., Harrington, J. y McChargue, D. (2008). Child sexual abuse, dissociation, and alcohol: implications of chemical dissociation via blackouts among college women. The American Journal of drugs and alcohol abuse, 34, 277-284, doi: 10.1080/00952990802013441.

Konstenius, M., Leifman, A., Van Emmerik, K., Van de Glind, G., Franck, J., Moggi, F., Ramos, J., Levin, F., Jan Carpentier, P., Skutle, A., Torild, E., Kaye, S., Demetrovics, Z., Barta, C., Auriecomb, M., Fatséas, M., Johnson, B., Faraone, S., Allsop, S., Carruthers, S., Schowvers, R., Verspreet, S., Dom, G., Koeter, M. y Van Den Brink, W. (2017). Childhood trauma exposure in substance use disorder patients with and without ADHD. Addictive Behaviors, 65, 118-124.

Krause, M. (1995). La investigación cualitativa: Un campo de posibilidades y desafios. Temas de educación, 15, 19-39.

Langeland, W., Draijer, N. y Van Den Brink, W. (2002). Trauma and dissociation in treatment-seeking alcoholics: Towards a resolution of inconsistent findings. Comprehensive psychiatry, 43 (3), 195-20, doi: 10.1053/comp.2002.32350.
Lyssenko, L., Schmahl, C., Bockhacker, L., Vonderlin, R., Bohus, M. y Kleindienst, N. (2017). Dissociation in psychiatric disorders: A meta-analysis of studies using the dissociative experiences scale. American Journal of Psychiatry, 175 (1), doi: 10.1176/ appi.ajp.2017.17010025.

Lythgoe, E. (2018). La memoria impedida en Paul Ricoeur. Kriterion, 141, 849-865.

Martínez-Salgado, C. (2012). El muestreo en investigación cualitativa: Principios básicos y algunas controversias. Ciência y Saúde Coletiva, 17 (3), 613-619.

Mau, M., Muller, A., Kaya-Roessler. (2018). Alcohol relapse and near-relapse experiences show that relapse models need to be updated. Alcoholism Treatment Quarterly, doi: 10.1080/07347324.2018.1532775

Mergler, M., Driessen, M., Havemann-Reinecke, U., Wedekind, D., Lüdecke, C., Ohlmeier, M., Chodzinski, C., Teunißen, S., Weirich, S., Schläfke, D., Renner, W., Schäfer, I., Koesters, G., Dette, C., Reis, O., Sylvester, E., Hoppe, M., Stuppe, M., Broese, T., Schneider, U. y Pletke, C. (2017). Relationships between a dissociative subtype of PTSD and clinical characteristics in patients with substance use disorders. Journal of Psychoactive Drugs, doi: 10.1080/02791072.2017.1296209.

Merriam, S. (2009). Qualitative research: a guide to design and implementation. Jossey-Bass.

Moustakas, C. (1994). Phenomenological research methods. Sage.

Najavits, L. y Walsh, M. (2012). Dissociation, PTSD, and substance abuse: an Empirical Study. Journal of trauma y Dissociation. 13: 115-126, doi: 10.1080/15299732.2011.608781.

Odland, B., Asplund, K. y Nergaard, T. (2015). Drinking resumption: Problematic alcohol use relapse after rehabilitation. A phenomenological hermeneutical perspective. Scandinavian journal of caring sciences 29 (4).

Olivares, F., Vargas, E., Fuentes, C., Martínez-Pernía, D. y CanalesJohnson, A. (2015). Neurophenomenology revisited: second-person methods for the study of human consciousness. Frontiers in psychology, 29(6), 673. Recuperado de: https://www.researchgate. net/publication/277331648_Neurophenomenology_revisited_second-person_methods_for_the_study_of_human_consciousness.

OMS. (2008). Glosario de términos de alcohol y drogas. Ministerio de sanidad y consumo. Centro de Publicaciones.

Perkowska, M. (2003). Memorias de un mundo perdido: rememoración y evocation en Fuegia, de Eduardo Belgrano Rawson. Cahiers du CRICCAL, 30 (1), 83-90.

Petitmengin, C. (2006). Describing one's subjective experience in the second person: An interview method for the science of consciousness. Phenomenology and the Cognitive Sciences, 5 (3-4), 229-269.

Petitmengin, C., Bitbol, M. y Ollagnier, M. (2015). Toward a science of lived experience. Intellecta, 64, 57-76.

Schäfer, I., Reininghaus, U., Langeland, W., Voss, A., Zieger, N., Haasen, C. y Karow, A. (2007). Dissociative symptoms and alcohol-dependent patients: associations with childhood trauma and substance abuse characteristics. Comprehensive Psychiatry, 48, 539-541, doi: 10.1016/j.comppsych.2007.05.013 
Silva-Mack, V., Araya-Véliz, C., Martínez-Pernía, D., y Arístegui Lagos, R. (2018). La experiencia de estar presente en meditadores zen: una aproximación fenomenológica. Límite (Arica), 13 (43), 29-39.

Tamar-Gurol, D., Sar, V., Karadag, F., Evren, C. y Karagoz, M. (2008). Chilhood emotional abuse, dissociation, and suicidality among patients with drug dependency in turkey. Psychiatry and Clinical Neurosciences, 62, 540-547, doi: 10.111/j.1440-1819.2008.01847.x
Van Den Bosch, L., Verheul, R., Langeland, W. y Van Den Brik, W. (2003). Trauma, Dissociation, and posttraumatic stress disorder in female borderline patients with and without substance abuse problems. Australian and New Zealand Journal of Psychiatry, 37, 549-555.

Van Den Brink, W. (2015). Substance use disorders, trauma, and PTSD. European Journal of Psychotraumatology, 6.

WHO (2018). Global status report on alcohol and health. Recuperado de https://apps.who.int/iris/bitstream/handle/106 65/274603/9789241565639-eng.pdf?ua=1. 